BMJ Open

Diabetes

Research

\& Care

\title{
Impact of undiagnosed type 2 diabetes and pre-diabetes on severity and mortality for SARS-CoV-2 infection
}

Arsenio Vargas-Vázquez, ${ }^{1,2}$ Omar Yaxmehen Bello-Chavolla (i) , ${ }^{3}$ Edgar Ortiz-Brizuela, ${ }_{4}^{4}$ Alejandro Campos-Muñoz, ${ }^{1}$ Roopa Mehta, ${ }^{1,5}$ Marco Villanueva-Reza, ${ }^{4}$ Jessica Paola Bahena-López, ${ }^{2}$ Neftali Eduardo Antonio-Villa (1) ,1,2,3 María Fernanda González-Lara, ${ }^{4}$ Alfredo Ponce de León, ${ }^{4}$ Jose Sifuentes-Osornio, ${ }^{6}$ Carlos Alberto Aguilar-Salinas (1) ${ }^{1,5}$

To cite: Vargas-Vázquez A, Bello-Chavolla OY, Ortiz-Brizuela E, et al. Impact of undiagnosed type 2 diabetes and pre-diabetes on severity and mortality for SARS-CoV-2 infection. BMJ Open Diab Res Care 2021;9:e002026. doi:10.1136/ bmjdrc-2020-002026

Received 19 November 2020 Revised 25 January 2021 Accepted 31 January 2021
Check for updates

(C) Author(s) (or their employer(s)) 2021. Re-use permitted under CC BY-NC. No commercial re-use. See rights and permissions. Published by BMJ.

For numbered affiliations see end of article.

Correspondence to Dr Carlos Alberto AguilarSalinas;

caguilarsalinas@yahoo.com

\section{ABSTRACT}

Introduction Diabetes and hyperglycemia are risk factors for critical COVID-19 outcomes; however, the impact of pre-diabetes and previously unidentified cases of diabetes remains undefined. Here, we profiled hospitalized patients with undiagnosed type 2 diabetes and pre-diabetes to evaluate its impact on adverse COVID-19 outcomes. We also explored the role of de novo and intrahospital hyperglycemia in mediating critical COVID-19 outcomes. Research design and methods Prospective cohort of 317 hospitalized COVID-19 cases from a Mexico City reference center. Type 2 diabetes was defined as previous diagnosis or treatment with diabetes medication, undiagnosed diabetes and pre-diabetes using glycosylated hemoglobin ( $\mathrm{HbA1C}$ ) American Diabetes Association (ADA) criteria and de novo or intrahospital hyperglycemia as fasting plasma glucose (FPG) $\geq 140 \mathrm{mg} / \mathrm{dL}$. Logistic and Cox proportional regression models were used to model risk for COVID-19 outcomes.

Results Overall, 159 cases (50.2\%) had type 2 diabetes and 125 had pre-diabetes (39.4\%), while $31.4 \%$ of patients with type 2 diabetes were previously undiagnosed. Among $20.0 \%$ of pre-diabetes cases and $6.1 \%$ of normalrange $\mathrm{HbA} 1 \mathrm{c}$ had de novo hyperglycemia. FPG was the better predictor for critical COVID-19 compared with HbA1c. Undiagnosed type 2 diabetes (OR: $5.76,95 \% \mathrm{Cl}$ 1.46 to 27.11 ) and pre-diabetes (OR: $4.15,95 \% \mathrm{Cl} 1.29$ to 16.75) conferred increased risk of severe COVID-19. De novo/intrahospital hyperglycemia predicted critical COVID-19 outcomes independent of diabetes status. Conclusions Undiagnosed type 2 diabetes, pre-diabetes and de novo hyperglycemia are risk factors for critical COVID-19. HbA1c must be measured early to adequately assess individual risk considering the large rates of undiagnosed type 2 diabetes in Mexico.

\section{INTRODUCTION}

Identification of high-risk individuals with COVID-19isusefultopreventcriticaloutcomes. Several studies have reported higher risk for severe COVID-19 and mortality in patients with type 2 diabetes mellitus (T2DM), obesity, hypertension, cardiovascular disease and

\section{Significance of this study}

What is already known about this subject?

- Patients with cardiometabolic diseases are at higher risk for adverse COVID-19 outcomes.

- Fasting plasma glucose levels and $\mathrm{HbA1c}$ have been identified as independent predictors for COVID-19 severity and mortality.

- What is the impact of pre-diabetes and undiagnosed type 2 diabetes on COVID-19 severity and mortality?

What are the new findings?

- Fasting plasma glucose is a better predictor for COVID-19 severity and mortality compared with HbA1c.

- Patients with pre-diabetes or undiagnosed type 2 diabetes are at higher risk for severe COVID-19.

- De novo hyperglycemia in patients with $\mathrm{HbA} 1 \mathrm{C}$ $<6.5 \%$ is an independent predictor for severe COVID-19.

\section{How might these results change the focus of} research or clinical practice?

- Triaging patients without diagnosed diabetes using $\mathrm{HbA1c}$ is needed to identify high-risk individuals.

other non-transmissible diseases. ${ }^{1}{ }^{2}$ Furthermore, fasting plasma glucose (FPG) levels and glycosylated hemoglobin (HbA1c) have been identified as independent predictors for COVID-19 severity and mortality. ${ }^{3}$ Similarly, mechanistic studies have demonstrated that chronic hyperglycemia is associated with immune dysregulation and susceptibility to severe lung disease in MERS-CoV (Middle East respiratory syndrome-related coronavirus) infection. ${ }^{5}$ Therefore, HbAlc levels could be associated with adverse outcomes from infectious diseases in patients with and without diabetes, including for COVID-19. ${ }^{5}$ 
Previous studies have evaluated the effect of preadmission glycemic control in patients with diabetes and COVID-19, concluding that higher preadmission levels of glucose were associated with higher morbidity and mortality. However, there are some studies that have shown that higher levels of HbAlc are associated with disease progression and mortality, while few studies have not. ${ }^{6-9}$ These results highlight the relevant role of diabetes treatment regimen and glycemic control prior to COVID-19 infection and their association with poor outcomes. In Mexico, the COVID-19 pandemic has resulted in increased mortality rates due to the high burden of cardiometabolic diseases, which facilitate the development of severe COVID-19. ${ }^{10}$ Notably, a large proportion of cases with T2DM in Mexico are unaware of their diagnosis ${ }^{11}$; these cases often present with severe glycemic imbalance and possibly undiagnosed chronic diabetes complications that complicate management and likely increase vulnerability to severe infection. ${ }^{12}$ Despite increasing evidence on the role of diabetes-specific risk factors for severe COVID-19 in T2DM, limited available evidence propose that undiagnosed T2DM is associated with poor COVID-19 outcomes compared with previously diagnosed T2DM, and the role of pre-diabetes (PreDM) on COVID-19 severity and mortality has not been reported. ${ }^{13-16}$ Here, we evaluated hospitalized patients with undiagnosed T2DM and PreDM to evaluate its impact on adverse COVID-19 outcomes. Similarly, we explored the possible mediating effect of hyperglycemia on severity and mortality for SARS-CoV-2 infection.

\section{MATERIAL AND METHODS \\ Study population}

We conducted an analysis of patients with complete clinical information of patients aged $\geq 18$ years from 16 March to 1 July 2020 whom had been hospitalized confirmed SARS-CoV-2 infection using real-time RT-PCR and had available HbAlc measurement at the point of admission at the Instituto Nacional de Ciencias Médicas y Nutrición Salvador Zubirán, a COVID-19 reference center in Mexico City. Informed consent was waived due to the nature of the study.

\section{Clinical information and definition of outcomes}

Information collected at the time of clinical triage included demographic variables, medical history, physical examination and biochemical measurements. Details regarding this cohort have been described elsewhere. ${ }^{17}$ Intensive care unit (ICU) admission was based on clinical judgment. Severe COVID-19 was determined as a composite of death, ICU admission or mechanical ventilation. Previously diagnosed T2DM was defined as a construct comprising previous medical diagnosis and/ or treatment with glucose-lowering agents. Undiagnosed T2DM was defined as HbA1c $\geq 6.5 \%$ and PreDM as HbAlc $5.70 \%-6.49 \%$ in cases without previously diagnosed PreDM according to the ADA criteria. ${ }^{18}$
De novo/intrahospital hyperglycemia was defined as FPG $\geq 140 \mathrm{mg} / \mathrm{dL}$ in cases without diabetes and normal HbA1c. ${ }^{19}$ Follow-up time was estimated from date of symptom onset to last follow-up (censoring) or death, whichever occurred first.

\section{Statistical analyses}

Proportions were compared using $\chi^{2}$ test and one-way analysis of variance or Kruskal-Wallis test depending on variable distribution. We evaluated prediction of admission to the ICU, requirement for invasive ventilation and/or severe COVID-19 using logistic regression analyses, goodness of fit was evaluated using the Hosmer-Lemeshow test. Logistic regression models were fitted for invasive ventilation and ICU admission due to incomplete data to estimate time-to-event models. For mortality, we fitted Cox proportional risk regression models estimating time from symptom onset up to death or censoring, whichever occurred first. Proportional risk assumptions were verified using Schönfeld residuals and visual inspection of time-varying effects. Model selection was carried out using minimization of the Bayesian Information Criterion (BIC).

We performed casual mediation analysis to investigate whether the presence of intrahospital hyperglycemia may act as a mediator of the risk conferred by inflammatory response to SARS-CoV-2 infection on severity and mortality. Mediation analyses were performed using mediation $\mathrm{R}$ package; to permit inference for the parameters estimated in the mediation models, we obtained $95 \%$ CIs using percentiles from bias-corrected accelerated non-parametric bootstrap $(\mathrm{B}=1000)$. Causal mediation analyses for mortality were tested using causally ordered model-based mediation analysis using beta coefficients extracted from Cox proportional risk regression models, as previously described. ${ }^{20} \mathrm{~A} p$ value $<0.05$ was considered as statistically significant. Analyses were performed using R software V.3.6.2.

\section{RESULTS}

Profiling undiagnosed type 2 diabetes and pre-diabetes in patients with SARS-CoV-2 infection

Among 917 inpatients evaluated from 16 March to 1 July 2020, we included 317 (33.86\%) inpatients with complete clinical information and HbAlc measurement on admission based on clinical judgment of the attending physician. Among them, 159 (50.2\%) had T2DM, 125 (39, 4\%) had PreDM and 33 (10.4\%) had normal-range HbAlc; however, $31.4 \%$ of patients with T2DM had undiagnosed T2DM. Similarly, $20 \%$ and $6.1 \%$ of patients with PreDM and cases with normal HbA1c had de novo hyperglycemia. Cases with undiagnosed T2DM, PreDM and cases with normal HbAlc were younger and had lower prevalence of hypertension and Charlson index compared with previously diagnosed T2DM; notably, undiagnosed T2DM cases had higher rates of ICU admission, severe infection and higher mortality $(\mathrm{p}<0.01)$. Previously 
diagnosed T2DM cases had higher FPG, HbA1c and triglycerides levels, while PreDM cases had higher AST levels and neutrophils-lymphocytes index. Undiagnosed T2DM cases had lower levels of albumin and $\mathrm{PaO}_{2} /$ fractional inspired oxygen $\left(\mathrm{FiO}_{2}\right)$ ratio but similar lactate levels than previously diagnosed cases. We observed no differences in other inflammation markers, viral load, time from symptom onset to clinical assessment or death between groups (table 1).

\section{Undiagnosed type 2 diabetes and pre-diabetes and their association with severe COVID-19 and mortality}

FPG was a better predictor for severity of infection (OR $1.89,95 \%$ CI 1.31 to 2.81 vs OR $1.36,95 \%$ CI 1.04 to 1.79 , $\triangle \mathrm{BIC}-39.1$ ) and mortality (HR $1.55,95 \%$ CI 1.19 to 2.01 vs HR $1.35,95 \%$ CI 1.04 to $1.74, \Delta \mathrm{BIC}-4.05$ ) compared with $\mathrm{HbAlc}$ adjusted for sex, age, body mass index (BMI) and the Charlson comorbidity index. Next, we observed that undiagnosed T2DM cases had higher odds of severe COVID-19 (OR 9.13, 95\% CI 3.21 to 30.64), compared with previously diagnosed T2DM (OR 5.10, 95\% CI 1.98 to 15.91 ) and PreDM (OR 3.85, 95\% CI 1.50 to 11.95). When adjusting for sex, age, BMI and number of comorbidities, undiagnosed T2DM cases still had higher odds of severe disease (OR 7.91, 95\% CI 2.59 to 28.07) when compared with previously diagnosed diabetes (OR 3.14, 95\% CI 1.12 to 10.31 ) and PreDM (OR 3.25, 95\% CI 1.20 to 10.46 , figure 1 ).

Compared with cases with normal HbAlc using Cox proportional hazard regression models, undiagnosed T2DM cases had a higher risk of mortality (HR 5.51, 95\% CI 1.28 to 23.81), followed by previously diagnosed T2DM (HR 4.98, 95\% CI 1.19 to 20.74) and PreDM (HR $3.35,95 \%$ CI 0.79 to 14.3 ) ; however, when adjusted by age, sex, BMI and comorbidities, only undiagnosed T2DM remained associated with mortality (HR 5.50, $95 \%$ CI 1.16 to 26.71 , figure 1 ). Finally, we evaluated the impact of de novo hyperglycemia in patients with HbAlc $\leq 6.4 \%$ and observed that these cases had higher odds of severe COVID-19 (OR 4.36, 95\% CI 1.53 to 13.67) but were not associated with mortality after adjustment for covariates. For subjects with T2DM, intrahospital hyperglycemia $(\geq 140 \mathrm{mg} / \mathrm{dL}$ ) was associated to a higher risk of adverse COVID-19 outcomes: severe infection (OR 3.80, $95 \%$ CI 1.56 to 9.85 ) and mortality (HR 2.75, 95\% CI 1.13 to 6.66$)$.

\section{Hyperglycemia mediates the effect of inflammation on} severity and lethality for SARS-CoV-2 infection

Next, we sought to investigate a mechanistic role for hyperglycemia on mediating adverse outcomes in severe COVID-19. First, we fitted lineal regression models to test the association of inflammatory markers with elevated glucose, and we observed that the levels of inflammatory markers on admission explain about $15 \%$ of glucose variability in COVID-19 patients with $\mathrm{HbAlc}<6.5 \%$ and less than $10 \%$ in patients with T2DM; these models were adjusted by sex, age, BMI and the Charlson comorbidity index (table 2).

Finally, to explore whether the hyperglycemia may act as mediator of the risk conferred by inflammatory response to SARS-CoV-2 outcomes, we developed two modelbased casual mediation analyses: hyperglycemia (FPG on admission) as a mediator of the effect of inflammatory response to infection $(\mathrm{C}$ reactive protein $(\mathrm{CRP})$ ) on severe COVID-19 and lethality. Also, we worked under the assumption that inflammatory response to SARS-CoV-2 infection is able to cause or worsen the hyperglycemia observed in these patients (figure 2). For the first model, both the direct effect of inflammatory response to infection (CRP) on severe COVID-19 $\left(\Delta_{\mathrm{E} \rightarrow \mathrm{Y}}=0.126,95 \%\right.$ CI 0.057 to 0.200 ) and the effect of inflammation that is mediated by hyperglycemia were statistically significant $\left(\Delta_{\mathrm{E} \rightarrow \mathrm{MY}}=0.021,95 \%\right.$ CI 0.005 to 0.0 .050$)$. Overall, the effect of inflammatory response to infection assessed with CRP on severe COVID-19 is modified by hyperglycemia, accounting for $14.7 \%$ of the effect of inflammation on severe COVID-19. Interestingly, when we stratified by T2DM diagnosis, we found that the effect mediated by hyperglycemia increased to $20.1 \%$ in patients without diabetes and to $16.0 \%$ in diabetes.

The second model-based mediation analysis focused on COVID-19 lethality. Using causally ordered mediating Cox models, we observed that both the direct effect of inflammatory response on lethality $\left(\Delta_{\mathrm{E} \rightarrow \mathrm{Y}}=1.518\right.$, $95 \%$ CI 1.202 to 1.919) and the indirect effect mediated by hyperglycemia $\left(\Delta_{\mathrm{E} \rightarrow \mathrm{MY}}=1.385,95 \%\right.$ CI 1.102 to 1.738$)$ were significant, representing $41.9 \%$ of the total effect of inflammatory response on lethality. When we stratified by T2DM, the mediating effect remained representing $43.0 \%$ of the risk conferred by inflammation in cases without diabetes and $40.1 \%$ for cases T2DM (table 3 ). All these models were adjusted by sex, age, BMI and Charlson index.

\section{DISCUSSION}

Here, we present a comprehensive report of COVID-19 cases with undiagnosed T2DM and PreDM and show that detection of undiagnosed T2DM and PreDM identifies subjects at higher risk of complications who may benefit from early triaging with $\mathrm{HbA1c}$ measurements to potentially reduce these complications. We also report a higher prevalence of cases with undiagnosed T2DM when compared with those observed during the influenza H1N1 pandemic and Mexican population..$^{21} 22$ Our data confirm observations regarding the association of diabetes with increased risk of severe COVID-19 and the predictive ability of FPG and HbA1c to predict complications. ${ }^{134}$ Given the large rate of undiagnosed T2DM and PreDM reported in our study, we support the recommendation that HbA1c measurement should be systematically measured to optimize COVID-19 management.

We also report that FPG is a better predictor for SARS-CoV-2 severity and mortality compared with 
Table 1 Baseline characteristics of the patient with SARS-CoV-2 according to glycosylated hemoglobin categories

\begin{tabular}{|c|c|c|c|c|c|}
\hline \multirow[b]{2}{*}{ Parameter } & \multicolumn{4}{|c|}{ Glycosylated hemoglobin (\%) } & \multirow[b]{2}{*}{ P value } \\
\hline & $<5.7 \%(\mathrm{n}=33)$ & Pre-diabetes $(n=125)$ & T2DM (n=109) & U-T2DM $(n=50)$ & \\
\hline Sex (male) & $26(78.8)$ & $80(64.0)$ & $76(69.7)$ & $25(50.0)$ & 0.031 \\
\hline Age (years) & $45.0(34.0-50.0)$ & $50.0(42.5-61.0)$ & $57.0(47.5-64.5)$ & $52.0(44.0-62.0)$ & $<0.001$ \\
\hline BMI $\left(\mathrm{kg} / \mathrm{m}^{2}\right)$ & $29.0(26.1-31.7)$ & $29.7(27.0-33.9)$ & $30.1(26.7-33.8)$ & $30.4(26.0-33.9)$ & 0.434 \\
\hline Obesity (\%) & $11(34.4)$ & $57(45.6)$ & $53(48.6)$ & $26(52.0)$ & 0.778 \\
\hline Hypertension (\%) & $6(18.2)$ & $31(24.8)$ & $51(46.8)$ & $13(26)$ & 0.001 \\
\hline CKD (\%) & $1(3)$ & $2(1.6)$ & $7(6.4)$ & $1(2.0)$ & 0.214 \\
\hline CVD (\%) & $2(6.3)$ & $2(1.6)$ & $9(8.3)$ & $4(8.0)$ & 0.111 \\
\hline Smoke (\%) & $8(24.7)$ & $22(17.6)$ & $24(22.6)$ & $7(14.0)$ & 0.494 \\
\hline Charlson index & $0(0-0.5)$ & $1(0-2)$ & $2(1-3)$ & $1(0-2)$ & $<0.001$ \\
\hline T2D treatment (\%) & $0(0)$ & $0(0)$ & $103(94.5)$ & $0(0)$ & $<0.001$ \\
\hline Metformin (\%) & $0(0)$ & $0(0)$ & $80(73.4)$ & $0(0)$ & $<0.001$ \\
\hline Insulin (\%) & $0(0)$ & $0(0)$ & 33 (30.3) & $0(0)$ & $<0.001$ \\
\hline $\begin{array}{l}\text { Time from symptom onset to } \\
\text { clinical assessment (days) }\end{array}$ & $8.0(6.5-9.5)$ & $7.0(5.0-10.0)$ & $7.0(4.0-10.0)$ & $7.0(6.0-12.0)$ & 0.499 \\
\hline Pneumonia (\%) & 25 (100.0) & $99(99.0)$ & 87 (98.7) & 35 (100.0) & 0.884 \\
\hline IMV (\%) & $5(15.2)$ & $26(20.8)$ & $24(22.0)$ & $13(26.0)$ & 0.695 \\
\hline ICU (\%) & $5(15.2)$ & $51(40.8)$ & $52(47.7)$ & $31(62.0)$ & $<0.001$ \\
\hline Severe infection (\%) & $5(15.2)$ & $51(40.8)$ & $52(47.7)$ & $31(62.0)$ & $<0.001$ \\
\hline $\begin{array}{l}\text { Time from symptom onset to } \\
\text { death (days) }\end{array}$ & $16.0(13.0-21.0)$ & $16(12.0-22.0)$ & $17(12.0-22.0)$ & 15.5 (12.0-26.0) & 0.994 \\
\hline Death (\%) & $2(6.1)$ & 27 (21.6) & 34 (31.2) & $18(36.0)$ & 0.006 \\
\hline Temperature $\left({ }^{\circ} \mathrm{C}\right)$ & $36.8(36.4-37.9)$ & $37.0(36.4-37.8)$ & $37.0(36.4-37.5)$ & $37.0(36.5-37.5)$ & 0.676 \\
\hline Respiratory rate & $26.0(24.0-31.0)$ & $28.0(24.0-35.0)$ & $30.0(25.5-36.0)$ & $30.0(26.5-36.0)$ & 0.093 \\
\hline $\mathrm{O}_{2}$ saturation $(\%)$ & 87 (81.0-89.0) & $82.5(70.0-88.0)$ & $82.5(70.0-87.0)$ & $83.0(60.7-85.0)$ & 0.011 \\
\hline $\mathrm{MAP}(\mathrm{mm} \mathrm{Hg})$ & 94.6 (86.5-100.0) & 91.8 (83.3-100.5) & 92.6 (85.3-100.9) & $94.6(86.0-101.0)$ & 0.802 \\
\hline Glucose (mg/dL) & $104.0(98.0-118.0)$ & $114.0(100.5-132.0)$ & 206 (141.0-311.5) & $181.5(130.7-271.7)$ & $<0.001$ \\
\hline HbA1c (\%) & $5.5(5.4-5.6)$ & $6.0(5.8-6.2)$ & $9.5(7.7-11.1)$ & $7.3(6.7-9.2)$ & $<0.001$ \\
\hline $\mathrm{HbA} 1 \mathrm{c} \leq 7.0 \%$ & $33(100)$ & $125(100)$ & $21(19.3)$ & $21(42.0)$ & $<0.001$ \\
\hline Triglycerides (mg/dL) & 125.5 (106.2-159.0) & $152.0(117.0-194.5)$ & $173.5(141.5-235.7)$ & $164.0(112.0-204.0)$ & $<0.001$ \\
\hline TyG index & $8.8(8.6-9.0)$ & $9.1(8.8-9.3)$ & $9.8(9.3-10.4)$ & $9.4(9.0-10.1)$ & $<0.001$ \\
\hline ALT (UI/L) & $38.0(26.6-55.0)$ & $40.5(26.4-64.0)$ & $33.0(20.5-54.2)$ & $34.2(25.3-63.4)$ & 0.110 \\
\hline AST (UI/L) & $40.5(26.8-53.3)$ & $49.9(35.4-68.4)$ & $39.0(27.0-63.9)$ & $42.8(27.8-56.2)$ & 0.043 \\
\hline Albumin (mg/dL) & $3.9(3.4-4.0)$ & $3.6(3.3-3.8)$ & $3.5(3.2-3.9)$ & $3.3(3.2-3.8)$ & 0.002 \\
\hline CRP & $14.4(4.7-23.2)$ & $16.3(9.1-25.0)$ & $16.3(9.1-25.6)$ & $14.7(9.1-23.9)$ & 0.601 \\
\hline $\begin{array}{l}\text { Lactate deshydrogenase } \\
\text { (UI/L) }\end{array}$ & $348.0(301.0-426.2)$ & $420.0(317.0-548.0)$ & $383.0(302.0-486.0)$ & $412.5(319.7-529.7)$ & 0.059 \\
\hline Fibrinogen & $700.0(542.0-854.0)$ & $752.0(605.5-896.0)$ & $734.0(577.0-896.0)$ & $647.0(481.2-872.7)$ & 0.286 \\
\hline Lactate (mmol(L) & $1.2(0.9-1.5)$ & $1.5(1.1-2-0)$ & $1.6(1.2-2.1)$ & $1.6(1.1-2.5)$ & 0.008 \\
\hline $\mathrm{PaO}_{2} / \mathrm{FiO}_{2}$ ratio & $242.3(192.8-276.0)$ & $197.0(124.0-250.0)$ & $181.0(95.5-248.5)$ & $160.0(99.3-232.2)$ & 0.003 \\
\hline Ferritin (ng/mL) & 627.8 (334.3-1108.8) & 649.4 (310.3-1021.4) & 766.4 (393.1-1233.9) & 564.1 (274.7-909.8) & 0.267 \\
\hline Neutrophils-lymphocytes index & $7.9(4.3-18.1)$ & $11.2(6.4-18.1)$ & $8.6(5.6-12.7)$ & $8.8(5.7-15.5)$ & 0.091 \\
\hline $\begin{array}{l}\text { RT-PCR CT } \\
\text { Threshold }\end{array}$ & $25.0(22.7-30.2)$ & $26.0(22.0-29.0)$ & $25.0(22.0-29.0)$ & $27.0(23.0-31.0)$ & 0.667 \\
\hline
\end{tabular}

Data are expressed as mean $( \pm S D)$ or median $(I Q R)$ for quantitative variables and frequencies and percentage for qualitative variables. ALT, alanine aminotransferase; AST, aspartate aminotransferase; BMI, body mass index; CKD, chronic kidney disease; CRP, C reactive protein; CVD, cardiovascular disease; $\mathrm{FiO}_{2}$, fractional inspired oxygen; $\mathrm{HbA1c}$, glycosylated hemoglobin; ICU, intensive care unit; IMV, invasive mechanical ventilation; MAP, mean arterial pressure; $\mathrm{PaO}_{2}$, partial pressure of oxygen; T2DM, type 2 diabetes mellitus; TyG, triglycerides and glucose index; $\mathrm{U}$ T2DM, undiagnosed type 2 diabetes mellitus. 
A
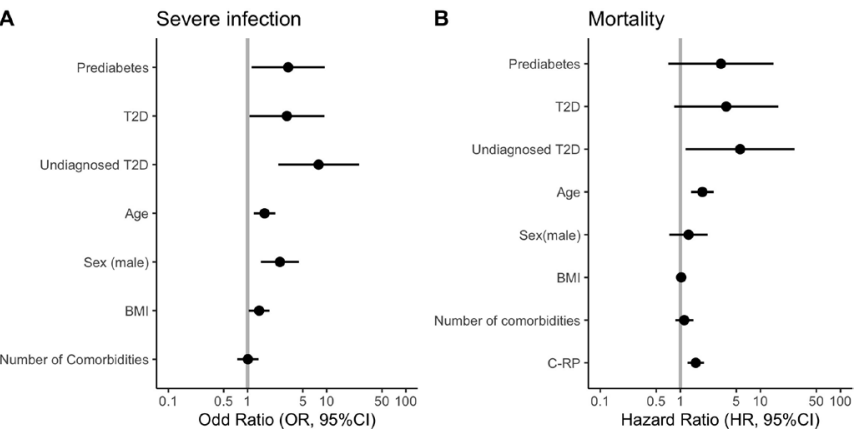

Figure 1 Logistic regression $(A)$ and Cox proportional regression (B) analysis to evaluated COVID-19 outcomes. $\mathrm{BMI}$, body mass index; C-RP, C reactive protein; T2DM, type 2 diabetes mellitus.

HbA1c. Although HbA1c reflects average glycemia over approximately 2-3 months and is the primary tool for assessing glycemic control, it also holds strong predictive value for diabetes complications and mortality. ${ }^{23}$ HbA1c does not provide a measure of glycemic variability or hypoglycemia as it frequently occurs during acute illness. ${ }^{1924}$ In addition, a significant interaction between HbA1c and glucose levels in critically ill patients has been reported. Therefore, the relationship between HbAlc and mortality changes according to the glucose levels. ${ }^{25}$ Similarly, several studies had showed that glycemic variability conferred a strong and independent risk of poor outcomes in heterogeneous population of critical ill patients. ${ }^{2627}$ Since glucose variability is not fully captured during acute illness by HbAlc, glucose levels at admission during an infectious process might confer an improved predictive value, as shown by our results.

In undiagnosed T2DM cases, HbA1c probably indicates pre-existing diabetes aggravated by COVID-19 leading to metabolic complications, and the dysglycemia observed in most cases might be explained by an adaptive metabolic response to critical illness and inflammation that involves neuroendocrine and immune pathways

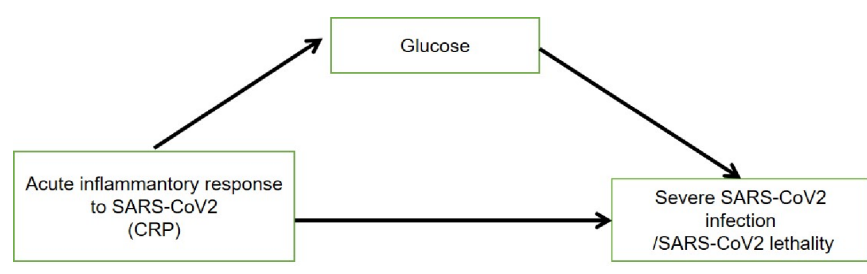

Figure 2 Causal mediation mechanism proposing the role of elevated plasma glucose levels on mediating the effect of acute inflammation on adverse SARS-CoV-2 outcomes explores in causally ordered mediation analyses. CRP, C reactive protein.

leading to transient insulin resistance and increased hepatic glucose production. ${ }^{28}{ }^{29}$ However, cases with de novo hyperglycemia identified during hospital stay must be followed up to identify subjects with transient hyperglycemia after recovery. This is relevant since during the SARS epidemic, it was observed that most hospitalized patients who were diagnosed with T2DM based on this criterion recovered completely after a 3 -year follow-up. ${ }^{30}$ Smith et al reported a prevalence of $17.1 \%$ for undiagnosed T2DM, which is higher in our study (33.4\%); similarly, we found that patients with undiagnosed T2DM had a lower $\mathrm{PaO}_{2} / \mathrm{FiO}_{2}$ ratio indicating a worse gas exchange in alveolar membrane, which might contribute to a higher risk of severe COVID-19 observed in this subgroup when compared with previously diagnosed or PreDM cases. In the same study, authors reported a PreDM prevalence of $20.4 \%,{ }^{31}$ which was also higher in our study $(39.4 \%)$. Increased prevalence of undiagnosed T2DM in younger patients support our prior findings that identified earlyonset T2DM as a COVID-19 risk factor, which could be further aggravated if undiagnosed as shown here. ${ }^{132}$ The relationship between glucose metabolism disruption and COVID-19 might be attributable to specific mechanisms of SARS-CoV-2 infection, pancreatic expression of ACE2 and its possible tropism for the pancreatic $\beta$ cell, and adaptive responses to critical illness that contributed

\begin{tabular}{llllll}
\hline Table 2 Linear regression models to test the association of glucose & with inflammatory markers & & \\
\hline & Parameter & $\boldsymbol{\beta}$ & $\mathbf{R}^{\mathbf{2}}$ & $\mathbf{9 5 \%} \mathbf{C l}$ & P value \\
\hline HbA1c $<6.5 \%$ & CRP & 0.191 & 0.162 & 0.069 to 0.313 & 0.002 \\
& LDH & 0.186 & 0.135 & 0.052 to 0.320 & 0.013 \\
& Ferritin & 0.212 & 0.165 & 0.076 to 0.348 & 0.002 \\
& Fibrinogen & 0.125 & 0.137 & -0.007 to 0.259 & 0.065 \\
& Neutrophils-lymphocytes index & 0.116 & 0.113 & -0.043 to 0.276 & 0.150 \\
HbA1c $26.5 \%$ and/or T2DM & CRP & 0.208 & 0.096 & 0.070 to 0.347 & 0.003 \\
& LDH & 0.123 & 0.063 & -0.029 to 0.277 & 0.116 \\
& Ferritin & 0.247 & 0.097 & 0.091 to 0.404 & 0.002 \\
& Fibrinogen & 0.153 & 0.092 & 0.020 to 0285 & 0.024 \\
& Neutrophils-lymphocytes index & 0.152 & 0.072 & 0.015 to 0.289 & 0.030 \\
\hline
\end{tabular}

Adjusted by sex, age, BMI and Charlson index.

$\mathrm{BMI}$, body mass index; CRP, C reactive protein; HbA1c, glycosylated hemoglobin; LDH, lactic dehydrogenase; T2DM, type 2 diabetes mellitus. 
Table 3 Causal mediation analysis predicting the mediating effect of acute inflammatory response to SARS-CoV-2 (E) mediated by elevated fasting plasma glucose $(\mathrm{M})$ on severe SARS-CoV-2 and lethality $(\mathrm{Y})$

\begin{tabular}{llllll}
\hline $\begin{array}{l}\text { Causal mediation } \\
\text { model }\end{array}$ & $\begin{array}{l}\text { Outcome } \\
(\mathbf{Y})\end{array}$ & ACME & ADE & Total effect & $\begin{array}{l}\text { Proportion } \\
\text { mediated }\end{array}$ \\
\hline Model 1 & Severe SARS-CoV-2 & 0.032 & 0.154 & 0.186 & $17.3 \%$ \\
& infection & $(0.014-0.050)$ & $(0.09-0.22)$ & $(0.126-0.250)$ & $(7.5-32.0)$ \\
& SARS-CoV-2 lethality & 1.113 & 1.484 & 1.653 & $40.25 \%$ \\
& & $(1.039-1.216)$ & $(1.197-1.839)$ & $(1.320-2.073)$ & $(36.97-44.04)$ \\
Model 2 & Severe SARS-CoV-2 & 0.021 & 0.126 & 0.147 & $14.7 \%$ \\
& infection & $(0.005-0.050)$ & $(0.057-0.200)$ & $(0.080-0.220)$ & $(8.05-22.0)$ \\
& SARS-CoV-2 lethality & 1.096 & 1.385 & 1.518 & $41.92 \%$ \\
Model 3 & & $(1.024-1.199)$ & $(1.102-1.738)$ & $(1.202-1.919)$ & $(38.45-46.01)$ \\
& Severe SARS-CoV-2 & 0.032 & 0.126 & 0.158 & $20.1 \%$ \\
& infection & $(0.003-0.070)$ & $(0.027-0.240)$ & $(0.061-0.240)$ & $(2.74-64.0)$ \\
Model 4 & SARS-CoV-2 lethality & 1.068 & 1.323 & 1.413 & $43.03 \%$ \\
& & $(0.958-1.225)$ & $(0.888-1.970)$ & $(0.95-2.085)$ & $(37.03-50.10)$ \\
& Severe SARS-CoV-2 & 0.020 & 0.110 & 0.131 & $16.0 \%$ \\
& infection & $(0.001-0.050)$ & $(0.033-0.200)$ & $(0.052-0.230)$ & $(0.3-44.0)$ \\
\hline
\end{tabular}

Model 1: unadjusted; model 2: adjusted by sex, age, BMI, Charlson index and HbA1c; model 3: subjects without T2DM and adjusted by sex, age, BMI and Charlson index; model 4: subjects with T2DM and adjusted by sex, age, BMI and Charlson index.

ACME, average causal mediation effects; ADE, average direct effects; BMI, body mass index; HbA1c, glycosylated hemoglobin; T2DM, type 2 diabetes mellitus.

to onset or worsening of hyperglycemia in patients with SARS-CoV-2. ${ }^{28}$

Furthermore, our results propose a potential mechanistic approach to explain the role of hyperglycemia as a mediator of inflammatory response to SARS-CoV-2 and their outcomes. We found that the hyperglycemia and inflammatory response observed in COVID-19 are intrinsically interlinked and that this relationship modifies the effect of both phenomena on SARS-CoV-2 morbidity and mortality. Moreover, we showed that hyperglycemia acts as a mediator of the effect of inflammatory response to infection on COVID-19 severity and mortality in inpatients with and without T2DM. The relationship of hyperglycemia and intrahospital poor health outcomes has previously demonstrated in several studies but not completely. ${ }^{33}$ Several mechanisms explain the detrimental effects of short-term hyperglycemia, which include alteration of host cellular defences by affecting leukocyte function (phagocytosis, chemotaxis, respiratory burst and antimicrobial activity) leading to hospital infections and poor recovery. ${ }^{17}$ Similarly, acute hyperglycemia results in the activation of nuclear factor $\mathrm{kB}$, production of proinflammatory cytokines and oxidative stress that increase mitochondrial and vascular endothelial dysfunction. These phenomena could potentially explain the excess of the risk observed in inpatients with COVID-19 and concomitant hyperglycemia. ${ }^{34-36}$ However, further basic studies and clinical studies are needed to explore the effect of hyperglycemia as mediator on adverse COVID-19 outcomes.

Here, we assessed the impact of undiagnosed T2DM and PreDM in SARS-CoV-2 outcomes. Limitations of our study include selection bias given the status of our hospital as a COVID-19 reference center and nonassessment of cases with mild COVID-19, which does not allow for an accurate estimation of the impact of undiagnosed T2DM and PreDM in the course of SARS-CoV-2 infection. Similarly, since HbA1c was only measured in patients according to their attending physician's discretion, further evaluations would be required with wide and systematic measurements of HbAlc in patients with mild and severe COVID-19.

In conclusion, our results highlight the need to triage non-diabetes patients and identify high-risk individuals. Follow-up studies will be required to explore de novo hyperglycemia observed in COVID-19 and determine the possible development of new-onset diabetes. Our results support that systematic assessment of HbA1c in critically ill patients would be useful to detect previously undiagnosed diabetes and PreDM to further contextualize the impact of glycemic variability in the setting of severe COVID-19.

\section{Author affiliations}

${ }^{1}$ Unidad de Investigación de Enfermedades Metabólicas, Instituto Nacional de Ciencias Médicas y Nutrición Salvador Zubiran, Mexico City, Mexico

${ }^{2} \mathrm{MD} / \mathrm{PhD}$ (PECEM) Program, Facultad de Medicina, Universidad Nacional Autónoma de México, Mexico City, Mexico

${ }^{3}$ Dirección de Investigación, Instituto Nacional de Geriatría, Mexico City, Mexico ${ }^{4}$ Departamento de Infectología, Instituto Nacional de Ciencias Médicas y Nutrición Salvador Zubiran, Mexico City, Mexico

${ }^{5}$ Department of Endocrinology and Metabolism, Salvador Zubiran National Institute of Medical Sciences and Nutrition, Tlalpan, Mexico

${ }^{6}$ Dirección de Medicina, Instituto Nacional de Ciencias Médicas y Nutrición Salvador Zubiran, Tlalpan, Mexico 
Acknowledgements All authors approved the submitted version. All the authors would like to thank the staff of the Endocrinology and Metabolism Department for all their support. We are thankful to the study volunteers for all their work and support throughout the realization of the study. AVV, JPBL and NEAV are enrolled in the Plan de Estudios Combinados en Medicina (PECEM) programme at the Faculty of Medicine of UNAM; AVV and JPBL are supported by CONACyT.

Contributors Research idea and study design: AVV, OYBC and CAAS; data acquisition: EOB, MFGL, APL and JSO; data analysis/interpretation: AVV, OYBC, ACM, RM, JPBL, MVR and CAAS; statistical analysis: AVV and OYBC; manuscript drafting: AVV, OYBC, ACM, NEAV, RM, MVR, JPBL, NEAV and CAAS; supervision or mentorship: CAAS. Each author contributed important intellectual content during manuscript drafting or revision and accepts accountability for the overall work by ensuring that questions pertaining to the accuracy or integrity of any portion of the work are appropriately investigated and resolved.

Funding The authors have not declared a specific grant for this research from any funding agency in the public, commercial or not-for-profit sectors.

Competing interests None declared.

Patient consent for publication Not required.

Ethics approval All proceedings were approved by the Institutional Review Board Research and Ethics Committee at Instituto Nacional de Ciencias Médicas y Nutrición Salvador Zubirán (approval number INF-3333-20-21-1), and informed consent was waived due to the nature of the study.

Provenance and peer review Not commissioned; externally peer reviewed.

Data availability statement Data are available on reasonable request. Data are available from the corresponding author on reasonable request.

Open access This is an open access article distributed in accordance with the Creative Commons Attribution Non Commercial (CC BY-NC 4.0) license, which permits others to distribute, remix, adapt, build upon this work non-commercially, and license their derivative works on different terms, provided the original work is properly cited, appropriate credit is given, any changes made indicated, and the use is non-commercial. See: http://creativecommons.org/licenses/by-nc/4.0/.

ORCID iDs

Omar Yaxmehen Bello-Chavolla http://orcid.org/0000-0003-3093-937X

Neftali Eduardo Antonio-Villa http://orcid.org/0000-0002-6879-1078

Carlos Alberto Aguilar-Salinas http://orcid.org/0000-0001-8517-0241

\section{REFERENCES}

1 Bello-Chavolla OY, Bahena-López JP, Antonio-Villa NE, et al. Predicting mortality due to SARS-CoV-2: a mechanistic score relating obesity and diabetes to COVID-19 outcomes in Mexico. J Clin Endocrinol Metab 2020;105:2752-61. doi:10.1210/clinem/ dgaa346

2 Zhou Y, Yang Q, Chi J, et al. Comorbidities and the risk of severe or fatal outcomes associated with coronavirus disease 2019: a systematic review and meta-analysis. Int J Infect Dis 2020;99:47-56.

3 Yang JK, Feng Y, Yuan MY, et al. Plasma glucose levels and diabetes are independent predictors for mortality and morbidity in patients with SARS. Diabet Med 2006;23:623-8.

4 Wang Z, Du Z, Zhu F. Glycosylated hemoglobin is associated with systemic inflammation, hypercoagulability, and prognosis of COVID-19 patients. Diabetes Res Clin Pract 2020;164:108214.

5 Kulcsar KA, Coleman CM, Beck SE, et al. Comorbid diabetes results in immune dysregulation and enhanced disease severity following MERS-CoV infection. JCl Insight 2019;4:e131774. doi:10.1172/jci. insight.131774

6 Cariou B, Hadjadj S, Wargny M, et al. Phenotypic characteristics and prognosis of inpatients with COVID-19 and diabetes: the CORONADO study. Diabetologia 2020;63:1500-15.

7 Ling P, Luo S, Zheng X, et al. Elevated fasting blood glucose within the first week of hospitalization was associated with progression to severe illness of COVID-19 in patients with preexisting diabetes: a multicenter observational study. J Diabetes 2021;13:89-93.

8 Sathish T, Cao Y. What is the role of admission HbA1c in managing COVID-19 patients? J Diabetes 2021;13:273-5.

9 Liu L, Wei W, Yang K, et al. Glycemic control before admission is an important determinant of prognosis in patients with coronavirus disease 2019. J Diabetes Investig 2020. doi:10.1111/jdi.13431. [Epub ahead of print: 09 Oct 2020].
10 Bello-Chavolla OY, Antonio-Villa NE, Vargas-Vázquez A, et al. Profiling pre-symptomatic and asymptomatic cases with confirmed SARS-CoV-2 infection in Mexico City. medRxiv2020.

11 Bello-Chavolla OY, Rojas-Martinez R, Aguilar-Salinas CA, et al. Epidemiology of diabetes mellitus in Mexico. Nutr Rev 2017;75:4-12.

12 Lampasona V, Secchi M, Scavini M, et al. Antibody response to multiple antigens of SARS-CoV-2 in patients with diabetes: an observational cohort study. Diabetologia 2020;63:2548-58.

13 Fadini GP, Morieri ML, Boscari F, et al. Newly-diagnosed diabetes and admission hyperglycemia predict COVID-19 severity by aggravating respiratory deterioration. Diabetes Res Clin Pract 2020;168:108374

$14 \mathrm{Li} \mathrm{H}$, Tian S, Chen T, et al. Newly diagnosed diabetes is associated with a higher risk of mortality than known diabetes in hospitalized patients with COVID-19. Diabetes Obes Metab 2020;22:1897-906.

15 Sathish T, de Mello GT, Cao Y. Is newly diagnosed diabetes a stronger risk factor than pre-existing diabetes for COVID-19 severity? J Diabetes 2021:13:177-8.

16 Sathish T, Cao Y, Kapoor N. Newly diagnosed diabetes in COVID-19 patients. Prim Care Diabetes 2021:15:194

17 Ortiz-Brizuela E, Villanueva-Reza M, González-Lara MF, et al. Clinical and epidemiological characteristics of patients diagnosed with covid-19 in a tertiary care center in Mexico City: a prospective cohort study. Rev Invest Clin 2020;72:165-77.

18 American Diabetes Association. 2. Classification and Diagnosis of Diabetes: Standards of Medical Care in Diabetes-2021. Diabetes Care 2021;44:S15-33.

19 American Diabetes Association. 15. Diabetes Care in the Hospital: Standards of Medical Care in Diabetes-2021. Diabetes Care 2021;44:S211-20.

20 Cho S-H, Huang Y-T. Mediation analysis with causally ordered mediators using COX proportional hazards model. Stat Med 2019;38:1566-81.

21 Kumar A, Wong R, Ottenbacher KJ, et al. Prediabetes, undiagnosed diabetes, and diabetes among Mexican adults: findings from the Mexican health and aging study. Ann Epidemiol 2016;26:163-70.

22 Ruiz PLD, Bakken IJ, Håberg SE, et al. Higher frequency of hospitalization but lower relative mortality for pandemic influenza in people with type 2 diabetes. J Intern Med 2020;287:78-86.

23 American Diabetes Association. 6. Glycemic Targets: Standards of Medical Care in Diabetes-2021. Diabetes Care 2021;44:S73-84

24 Beck RW, Connor CG, Mullen DM, et al. The Fallacy of Average: How Using $\mathrm{HbA}_{1 \mathrm{c}}$ Alone to Assess Glycemic Control Can Be Misleading. Diabetes Care 2017;40:994-9.

25 Egi M, Bellomo R, Stachowski E, et al. The interaction of chronic and acute glycemia with mortality in critically ill patients with diabetes. Crit Care Med 2011;39:105-11.

26 Krinsley JS. Glycemic variability: a strong independent predictor of mortality in critically ill patients. Crit Care Med 2008;36:3008-13.

27 Ali NA, Krinsley JS, Preiser JC. Glucose variability in critically ill patients. In: Vincent J-L, ed. Intensive care medicine. New York, NY: Springer New York, 2009: 728-37.

28 Apicella M, Campopiano MC, Mantuano M, et al. COVID-19 in people with diabetes: understanding the reasons for worse outcomes. Lancet Diabetes Endocrinol 2020;8:782-92.

29 Márquez-Salinas A, Fermín-Martínez CA, Antonio-Villa NE. Adaptive metabolic and inflammatory responses identified using accelerated aging metrics are linked to adverse outcomes in severe SARS-CoV-2 infection. medRxiv2020.

30 Yang J-K, Lin S-S, Ji X-J, et al. Binding of SARS coronavirus to its receptor damages islets and causes acute diabetes. Acta Diabetol 2010;47:193-9.

31 Smith SM, Boppana A, Traupman JA, et al. Impaired glucose metabolism in patients with diabetes, prediabetes, and obesity is associated with severe COVID-19. J Med Virol 2020. doi:10.1002/ jmv.26227. [Epub ahead of print: 26 Jun 2020].

32 Bello-Chavolla OY, Antonio-Villa NE, Ortiz-Brizuela E, et al. Validation and repurposing of the MSL-COVID-19 score for prediction of severe COVID-19 using simple clinical predictors in a triage setting: the Nutri-CoV score. PLoS One 2020;15:e0244051.

33 Wang S, Ma P, Zhang S, et al. Fasting blood glucose at admission is an independent predictor for 28-day mortality in patients with COVID-19 without previous diagnosis of diabetes: a multi-centre retrospective study. Diabetologia 2020;63:2102-11.

34 dandona P. Vascular reactivity in diabetes mellitus. Endocrinología y Nutrición 2009;56:12-14.

35 Lontchi-Yimagou E, Sobngwi E, Matsha TE, et al. Diabetes mellitus and inflammation. Curr Diab Rep 2013;13:435-44.

36 Jafar N, Edriss H, Nugent K. The effect of short-term hyperglycemia on the innate immune system. Am J Med Sci 2016;351:201-11. 